\title{
Comparative evaluation of the prophylactic activity of a slow-release insecticide collar and a moxidectin spot-on formulation against Thelazia callipaeda infection in naturally exposed dogs in France
}

Charlotte Lechat ${ }^{1}$, Noémie Siméon ${ }^{2}$, Olivier Pennant ${ }^{3}$, Loïc Desquilbet ${ }^{4}$, Sabine Chahory ${ }^{5}$, Christophe Le Sueur ${ }^{6}$ and Jacques Guillot ${ }^{{ }^{*}}$

\begin{abstract}
Background: The relative efficacy of a collar containing 10\% imidacloprid and 4.5\% flumethrin (Seresto ${ }^{\oplus}$, Bayer HealthCare Animal Health) and a spot on formulation containing 10\% imidacloprid and 2.5\% moxidectin (Advocate ${ }^{\oplus}$, Bayer HealthCare Animal Health) was evaluated as a control measure to prevent canine thelaziosis in dogs in an endemic area of France.

Findings: Ninety-six privately-owned dogs were enrolled in the multicentre, controlled study. Before summer (the period of transmission by fruit flies), dogs were allocated to one of three groups: Group $A(n=36)$ - treated once with a collar containing 10\% imidacloprid and 4.5\% flumethrin; Group B $(n=33)$ - treated every month for 8 months with a spot-on containing imidacloprid $10 \%$ and moxidectin $2.5 \%$; and Group C $(n=27)$ - untreated control animals. Dogs were regularly subjected to ocular examination in order to assess Thelazia callipaeda infection. During the trial, T. callipaeda nematodes were detected in 12 (33\%) collared dogs (group A) whereas no eyeworm could be found in dogs who received a monthly spot on application of moxidectin (group B). In the control group, 8 (30\%) dogs became infected.

Conclusions: The monthly application of a spot on formulation containing $10 \%$ imidacloprid and $2.5 \%$ moxidectin was shown to be highly effective in preventing T. callipaeda infection in a population of dogs living in an endemic area in France. On the contrary, the slow-release collar tested in this study did not display any protection against canine thelaziosis.
\end{abstract}

Keywords: Thelazia callipaeda, Eyeworm, Prevention, Dog, France

\section{Findings}

\section{Background}

Thelazia callipaeda (Spirurida, Thelaziidae) is a nematode that lives in the conjunctival sac of domestic and wild carnivores, rabbits and humans causing mild to severe clinical signs (epiphora, conjunctivitis, keratitis and even corneal ulcers) [1]. In Europe, Thelazia callipaeda is transmitted by Phortica variegata (Diptera, Drosophilidae),

\footnotetext{
* Correspondence: jguillot@vet-alfort.fr

${ }^{1}$ Parasitology, Ecole nationale vétérinaire d'Alfort, Maisons-Alfort, France Full list of author information is available at the end of the article
}

a small secretophagous fly. This insect feeds on fruits, vegetables and also on lacrymal secretions of domestic animals and wildlife [2]. In the past two decades T. callipaeda infection was proved to be widespread among dogs and cats from northern (Aosta valley) to southern (Basilicata region) Italy [3,4]. Following the first descriptions in Italy, T. callipaeda has been increasingly reported in western France (Dordogne area) [5,6], Switzerland [7], Spain [8], and Portugal [9]. Recently, the first autochthonous cases of thelaziosis have been described in red foxes, dogs and a cat living in Bosnia and Herzegovina and Croatia [10]. The 
geographical expansion of T. callipaeda in previously nonendemic countries of Europe may be attributed to the dispersal of the infection with wild carnivores (especially red foxes), which are suitable hosts for this parasite and may easily move in neighbouring regions [11]. The growing number of cases of canine thelaziosis in Europe is also a consequence of the increased mobility of dogs and the absence of reliable preventive measures in pet carnivores. In a previous investigation, monthly administration of milbemycine oxime was shown to significantly reduce $T$. callipaeda infection rate in dogs in the field [12]. Milbemycine oxime is the only molecule, which has been registered in Europe for the treatment of canine thelaziosis. The molecule is used orally at $0.5 \mathrm{mg} / \mathrm{kg}$ and two administrations at one week interval are recommended. Recently, a polymer matrix collar containing a combination of $10 \%$ imidacloprid and $4.5 \%$ flumethrin (Seresto ${ }^{\circ}$, Bayer HealthCare Animal Health) has been licensed for use in dogs and cats. This collar conferred long-term protection against fleas and ticks [13]. It was successful in preventing the transmission of tick or flea-borne pathogens $[14,15]$ and a study recently demonstrated its efficacy in the prevention of infection by Leishmania infantum in dogs in a hyperendemic area in Italy [16].

The aim of this study was to determine whether application of the slow-release insecticide collar containing $10 \%$ imidacloprid and $4.5 \%$ flumethrin could reduce $T$. callipaeda transmission in dogs living in Dordogne, France. The prophylactic activity of a spot-on formulation containing moxidectin was also evaluated over the 9 month-study period (from May 2012 to January 2013).

\section{Methods}

\section{Study site and selection of the dogs}

Ninety-six dogs (46 males and 50 females, aged 11 months to 15.6 years and weighting 5.4 to $54.8 \mathrm{~kg}$ ) were enrolled in the present trial. They were privately owned dogs who lived in Dordogne, France. Before inclusion, informed owner consent was obtained. Two veterinary clinics participated to the selection and follow up of the animals. These clinics are located in Vergt and Notre Dame de Sanilhac, two small cities within a zone known to be endemic for T. callipaeda [6].

Dogs fulfilling the following inclusion criteria were enrolled in the study: dogs in normal general health, $\geq$ 7 weeks of age, $\geq 1 \mathrm{~kg}$, not treated with ectoparasiticidal products in the preceding months, and with a history of previous infection by T. callipaeda (diagnosed in 2011 by direct examination of nematodes by NS or OP).

\section{Study design}

In May 2012, included dogs were assigned to one of the three groups, A, B and C, using a random treatment allocation plan. One month before the day of inclusion (D0) a spot on formulation containing 10\% imidacloprid and 2.5\% moxidectin (Advocate ${ }^{\circ}$, Bayer HealthCare Animal Health) was administered to all the dogs in order to be sure that the animals were not infected by $T$. callipaeda at the beginning of the trial. Dogs were allocated to one of three groups (A, B and C). At D0, dogs were checked again for eyeworms and imidacloprid $10 \%$ + flumethrin $4.5 \%$ collars (Seresto ${ }^{\circ}$, Bayer HealthCare Animal Health) were fitted to $\operatorname{dogs}(\mathrm{n}=36)$ from group $\mathrm{A}$ on the basis of their body weight (i.e., small collar: $<8 \mathrm{~kg} / \mathrm{large}$ collar: $>$ $8 \mathrm{~kg})$. Dogs $(\mathrm{n}=33)$ from group B received a monthly dose of imidacloprid $10 \%$ and moxidectin $2.5 \%$ by spot-on dermal application (Advocate ${ }^{\circ}$, Bayer HealthCare Animal Health), in accordance with the dogs' body weight and following the label instructions. Dogs from group B were treated 8 times (from May to December 2012). Dogs ( $\mathrm{n}=27$ ) from group $\mathrm{C}$ were left untreated and served as controls. Dogs from group A and $\mathrm{C}$ were examined on days 0, 120 and 240 after inclusion. Dogs from group B were examined every month (just before the application of the spot on) until day 240. During the trial, owners were asked to present their dogs to the consultation as soon as ocular clinical signs occurred. For each dog and each follow-up, the presence of $T$. callipaeda was carefully investigated by ocular examination. Dogs were restrained with a muzzle and the conjunctival fornix inspected for the presence of nematodes using a sterile cotton swab. An approximate count of nematodes number was made for each eye. Nematodes were stored in $70 \%$ ethanol and sent to the Parasitology department of Veterinary College of Alfort, France for morphological identification according to Skrjabin et al. [17] and Otranto et al. [18]. Infected dogs were treated by the application of the moxidectin spot on formulation.

The use of other ectoparasiticides on dogs was not allowed throughout the study period. However, individual treatments (with a product without any repellent activity) were authorized when heavy tick or flea infestations occurred. Due to the nature of the investigational veterinary product (collar), blinding was not applicable.

\section{Statistical analysis}

The efficacy evaluation was based on the comparison of the incidence of $T$. callipaeda-infected dogs in the two treatment groups (A and B) versus the control group (C). Incidence was calculated as follows: number of dogs newly infected with T. callipaeda/(number of negative dogs initially enrolled - number of lost or dead dogs) $\times 100$.

Statistical associations were quantified using prevalence rates of T. callipaeda in each of the 3 groups, and were tested using the Chi-2, or Fisher exact (when necessary), statistical test. Factors associated with higher prevalence rates of $T$. callipaeda were also investigated using the Chi-2 or Fisher exact statistical test. Statistical analyses were performed using Epi-Info (Centers for 


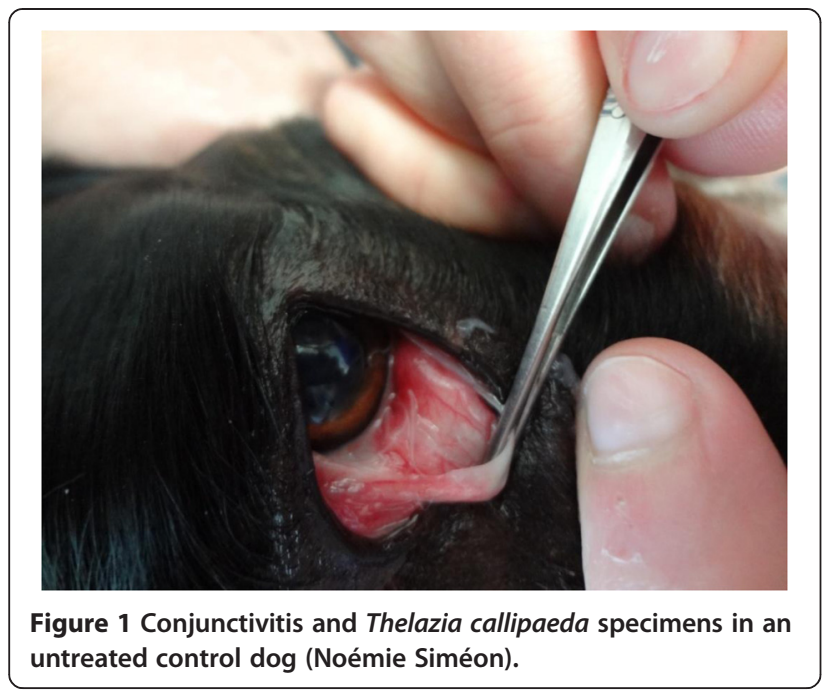

Disease Control and Prevention, USA; World Health Organization, Geneva, Switzerland). Significance level was reached if $\mathrm{p}$-values were $<0.05$.

\section{Results}

During the trial, $T$. callipaeda nematodes were detected in $12(33 \%)$ of the 36 collared dogs (group A) whereas no eyeworm could be found in the 33 dogs who received a monthly spot on application of moxidectin (group B). In the control group C, 8 (30\%) of the 27 dogs had eyeworms indicating that dogs living in the study area had been naturally exposed to T. callipaeda during the summer of 2012. There was no significant difference between the incidence of $T$. callipaeda infection in groups $\mathrm{A}$ and $\mathrm{C}$. As a consequence, the slow-release insecticide collar tested in this study did not provide any protection against canine thelaziosis. No signs suggestive of adverse reactions were recorded in treated dogs (either collared or treated with the spot on). Heavy tick or flea infestations never occurred during the trial. As a consequence, no additional insecticide or acaricide treatment was necessary.

One month before inclusion (in April 2012), out of the overall 96 dogs, 30 (31\%) were already infected by T. callipaeda. During the trial, 20 additional cases were detected. Seven dogs were infected twice (before inclusion and during the trial). In 12 cases, no clinical signs were detected. In other cases $(n=38)$, the following clinical signs were most frequently observed: conjunctival hyperemia (87\%), lacrimation (50\%), ocular mucopurulent discharge (24\%) and chemosis (11\%) (Figure 1).

The statistical analysis revealed that the frequencies of the variable parameters were not significantly different among groups (Table 1).

\section{Discussion}

The present study demonstrated that the monthly application of a spot on formulation containing imidacloprid and moxidectin was highly effective in preventing $T$. callipaeda infection in a population of dogs living in an endemic area in France. On the contrary, the slow-release collar tested in this study did not display any protection. To our knowledge, only two trials were performed in order to evaluate the prophylactic activity of antiparasitic drugs against $T$. callipaeda. Rossi et al. evaluated the efficacy of a single parenteral administration of moxidectin in naturally exposed dogs in northern Italy [19]. In June 2005, 31 dogs were injected subcutaneously with a

Table 1 Baseline characteristics of groups A (dogs treated with Seresto ${ }^{\circledR}$ ), B (dogs treated with Advocate ${ }^{\circledR}$ ) and $C$ (untreated dogs)

\begin{tabular}{|c|c|c|c|c|}
\hline Parameters & Group A $(n=36)$ Seresto $^{\oplus}$ & Group B $(n=33)$ Advocate ${ }^{\circledast}$ & Group C ( $n=27)$ & $P$ value \\
\hline Pure breed, n (\%) & $26(72.2 \%)$ & $26(78.8 \%)$ & $16(59.3 \%)$ & 0.25 \\
\hline \multicolumn{5}{|l|}{ Hair length, n (\%) } \\
\hline Short hair & $16(44.5 \%)$ & $11(33.3 \%)$ & 7 (25.9\%) & \multirow[t]{3}{*}{0.59} \\
\hline Medium hair & $12(33.3 \%)$ & $14(42.4 \%)$ & $14(51.9 \%)$ & \\
\hline Long hair & $8(22.2 \%)$ & $8(24.3 \%)$ & $6(22.2 \%)$ & \\
\hline Median age [IQR] (years) & $5.7[3.1-8.5]$ & $5.8[2.4-9.8]$ & $4.5[2.8-7.4]$ & 0.97 \\
\hline Male, n (\%) & $19(52.8 \%)$ & $13(39.4 \%)$ & $14(51.9 \%)$ & 0.48 \\
\hline Contact with other carnivores, n (\%) & $30(83.3 \%)$ & $25(75.8 \%)$ & $17(63 \%)$ & 0.18 \\
\hline Living in rural area (versus city), n (\%) & $35(97.2 \%)$ & $32(97 \%)$ & $25(92.6 \%)$ & 0.61 \\
\hline \multicolumn{5}{|l|}{ Type of living, n (\%) } \\
\hline Farm & $9(25 \%)$ & $7(21.2 \%)$ & $6(22.2 \%)$ & \multirow[t]{3}{*}{0.98} \\
\hline House & $26(72.2 \%)$ & $26(78.8 \%)$ & $21(77.8 \%)$ & \\
\hline Flat & $1(2.8 \%)$ & $0(0 \%)$ & $0(0 \%)$ & \\
\hline Median body weight [IQR] (kg) & $24.8[17.7-29.2]$ & $26.0[15.8-29.5]$ & 24.5 [19.3-34.1] & 0.80 \\
\hline
\end{tabular}

$\mathrm{IQR}$, Interquartile range. 
sustained-release moxidectin product (at the dose of $0.17 \mathrm{mg} / \mathrm{kg}$ ) and 32 dogs served as untreated controls. At the end of the trial in January 2006, none of the moxidectin-treated dogs had eyeworms whereas 11 (34\%) of the control dogs did. A similar investigation was made to evaluate the prophylactic efficacy of milbemycin oxime [12]. Thirty dogs were treated with oral milbemycin oxime monthly from June to November 2007 with the recommended dose rate for the prevention of heartworm disease and 30 dogs served as untreated controls. One dog in the treated group and 10 dogs in the control group became infected during the trial [12]. As macrocyclic lactones (like moxidectin and milbemycin oxime) have a limited effect on vectors (like the fruit fly $P$. variegata), the prophylactic efficacy of these drugs is accounted by their activity against $T$. callipaeda larvae a short time after they are deposited in the lacrymal secretions by a fruit fly. The therapeutic efficacy of the spot on formulation containing 10\% imidacloprid and $2.5 \%$ moxidectin was demonstrated in naturally infected dogs in Italy [20]. Elimination of T. callipaeda was obtained within 5 or 9 days after treatment.

The use of an insecticide that could limit the exposure of dogs to contaminating fruit flies is another prophylactic strategy. The use of collars impregnated with acaricidal/ repellent compounds has provided promising results, indicating that long-lasting protection of dogs against major canine vector-borne diseases can be achieved in most instances [14]. In the present study, the final incidence of thelaziosis in collared dogs and in control animals was not significantly different suggesting that the polymer matrix collar containing a combination of imidacloprid and flumethrin would not provide any protection against canine thelaziosis in field conditions. The amount of active ingredients released by the collar that reach the skin area where the fruit fly uses to land on and the short contact time are probably not sufficient to ensure a repellent activity against fruit flies or to kill them before they are able to feed and transmit $T$. callipaeda larvae.

\section{Conclusion}

As a conclusion, the prevention of canine thelaziosis is possible with a systemic anthelmintic molecule like moxidectin. For dogs living in endemic areas, the monthly administration of systemic macrocyclic lactones throughout the transmission season (summer) should be recommended. For pets spending no more than one month in endemic areas, a single treatment, usually soon after returning home, should be sufficient to assure complete protection.

\section{Competing interests}

C Le Sueur is an employee of Bayer HealthCare Animal Health, Puteaux, France. All authors voluntarily publish this article and have no personal interest in this trial other than publishing the scientific findings.

\section{Authors' contributions}

$J G$ and CLS conceived and designed the survey. NS and OP carried out the field survey. $C L$ monitored the study. $L D$ and $C L$ performed the statistical analysis of data. JG and $\mathrm{CL}$ drafted the first version of the manuscript. All authors have contributed to the revision of the manuscript and approved its final version.

\section{Author details}

${ }^{1}$ Parasitology, Ecole nationale vétérinaire d'Alfort, Maisons-Alfort, France. ${ }^{2}$ Veterinary Clinic, Notre Dame de Sanilhac, France. ${ }^{3}$ Veterinary Clinic, Vergt, France. ${ }^{4}$ Biostatistics and clinical epidemiology, Ecole nationale vétérinaire d'Alfort, Maisons-Alfort, France. ${ }^{5}$ Ophthalmology, Ecole nationale vétérinaire d'Alfort, Maisons-Alfort, France. ${ }^{6}$ Bayer HealthCare Animal Health, Puteaux, France.

Received: 17 October 2014 Accepted: 26 January 2015

Published online: 10 February 2015

\section{References}

1. Otranto D, Dantas-Torres F, Brianti E, Traversa D, Petrić D, Genchi C, et al. Vector-borne helminths of dogs and humans in Europe. Parasit Vectors. 2013;6:16.

2. Otranto D, Lia RP, Cantacessi C, Testini G, Troccoli A, Shen JL, et al. Nematode biology and larval development of Thelazia callipaeda (Spirurida, Thelaziidae) in the drosophilid intermediate host in Europe and China. Parasitology. 2005;131:847-55.

3. Rossi L, Bertaglia P. Presence of Thelazia callipaeda Railliet and Henry, 1910, in Piedmont, Italy. Parassitologia. 1989;31:167-72.

4. Otranto D, Ferroglio E, Lia RP, Traversa D, Rossi L. Current status and epidemiological observations of Thelazia callipaeda (Spirurida, Thelaziidae) in dogs, cats and foxes in Italy: a "coincidence" or a parasitic disease of the Old Continent? Vet Parasitol. 2003;116:315-25.

5. Dorchies P, Chaudieu G, Siméon LA, Cazalot G, Cantacessi C, Otranto D. First reports of autochthonous eyeworm infection by Thelazia callipaeda (Spirurida, Thelaziidae) in dogs and cat from France. Vet Parasitol. 2007;149:294-7.

6. Ruytoor $P$, Déan E, Pennant $O$, Dorchies $P$, Chermette $R$, Otranto D, et al. Ocular thelaziosis in dogs, France. Emerg Infect Dis. 2010;16:1943-5.

7. Malacrida F, Hegglin D, Bacciarini L, Otranto D, Nägeli F, Nägeli C, et al. Emergence of canine ocular Thelaziosis caused by Thelazia callipaeda in southern Switzerland. Vet Parasitol. 2008;157:321-7.

8. Miró G, Montoya A, Hernández L, Dado D, Vázquez MV, Benito M, et al. Thelazia callipaeda: infection in dogs: a new parasite for Spain. Parasit Vectors. 2011:4:148.

9. Rodrigues FT, Cardoso L, Coutinho T, Otranto D, Diz-Lopes D. Ocular thelaziosis due to Thelazia callipaeda in a cat from northeastern Portugal. J Feline Med Surg. 2012;14:952-4.

10. Hodžić A, Latrofa MS, Annoscia G, Alić A, Beck R, Lia RP, et al. The spread of zoonotic Thelazia callipaeda in the Balkan area. Parasit Vectors. 2014;30:352.

11. Otranto D, Dantas-Torres F, Mallia E, DiGeronimo PM, Brianti E, Testini G, et al. Thelazia callipaeda (Spirurida, Thelaziidae) in wild animals: report of new host species and ecological implications. Vet Parasitol. 2009;166:262-7.

12. Ferroglio E, Rossi L, Tomio E, Schenker R, Bianciardi P. Therapeutic and prophylactic efficacy of milbemycin oxime (Interceptor) against Thelazia callipaeda in naturally exposed dogs. Vet Parasitol. 2008;154:351-3.

13. Stanneck D, Rass J, Radeloff I, Kruedewagen E, Le Sueur C, Hellmann K, et al. Evaluation of the long-term efficacy and safety of an imidacloprid 10\%/ flumethrin $4.5 \%$ polymer matrix collar (Seresto ${ }^{\oplus}$ ) in dogs and cats naturally infested with fleas and/or ticks in multicentre clinical field studies in Europe. Parasit Vectors. 2012;5:66

14. Dantas-Torres F, Capelli G, Giannelli A, Ramos RA, Lia RP, Cantacessi C, et al. Efficacy of an imidacloprid/flumethrin collar against fleas, ticks and tickborne pathogens in dogs. Parasit Vectors. 2013;6:245.

15. Lappin MR, Davis WL, Hawley JR, Brewer M, Morris A, Stanneck D. A flea and tick collar containing 10\% imidacloprid and 4.5\% flumethrin prevents flea transmission of Bartonella henselae in cats. Parasit Vectors. 2013;6:26.

16. Brianti E, Gaglio G, Napoli E, Falsone L, Prudente C, Solari Basano F, et al. Efficacy of a slow-release imidacloprid (10\%)/flumethrin (4.5\%) collar for the prevention of canine leishmaniosis. Parasit Vectors. 2014;7:327.

17. Skrjabin KI, Sobolev AA, Ivashkin VM. Principles of Nematology, vol. IX. Spirurata of Animals and Man and the Disease Caused by Them. Part 4: 
Thelazioidea. Izdatel'sto Akademii Nauk SSSR. Washington, USA: Israel Program for Scientific Translations; 1967.

18. Otranto D, Lia RP, Traversa D, Giannetto S. Thelazia callipaeda (Spirurida, Thelaziidae) of carnivores and humans: morphological study by and scanning electron microscopy. Parassitologia. 2004;45:125-33.

19. Rossi L, Rigano C, Tomio E, Frassetto D, Ferroglio E. Use of sustained-release moxidectin to prevent eyeworm (Thelazia callipaeda) infection in dogs. Vet Rec. 2007:161:820-1.

20. Bianciardi P, Otranto D. Treatment of dog thelaziosis caused by Thelazia callipaeda (Spirurida, Thelaziidae) using a topical formulation of imidacloprid $10 \%$ and moxidectin 2.5\%. Vet Parasitol. 2005;129:89-93.

\section{Submit your next manuscript to BioMed Central} and take full advantage of:

- Convenient online submission

- Thorough peer review

- No space constraints or color figure charges

- Immediate publication on acceptance

- Inclusion in PubMed, CAS, Scopus and Google Scholar

- Research which is freely available for redistribution 